\title{
Kaposi Sarcoma NCI Grade 3
}

National Cancer Institute

\section{Source}

National Cancer Institute. Kaposi Sarcoma NCI Grade 3. NCI Thesaurus. Code C9414.

A Kaposi sarcoma with more than 15\% necrosis. 